\title{
Post COVID-19 Arterial Thromboembolism: A Clear and Present Danger
}

\author{
Bingwen Eugene Fan, MBBS, MRCP, MMED $1,2,3,4$ 이 \\ ${ }^{1}$ Department of Haematology, Tan Tock Seng Hospital, Singapore \\ ${ }^{2}$ Department of Laboratory Medicine, Khoo Teck Puat Hospital, \\ Singapore \\ ${ }^{3}$ Lee Kong Chian School of Medicine, Nanyang Technological \\ University, Singapore \\ ${ }^{4}$ Yong Loo Lin School of Medicine, National University of Singapore, \\ Singapore \\ ${ }^{5}$ Institute of Molecular and Cell Biology, Singapore
}

Semin Thromb Hemost 2022;48:112-114.

\author{
Christine Cheung, $\mathrm{PhD}^{3,5}$
}

Address for correspondence Bingwen Eugene Fan, MBBS, MRCP, MMED, Department of Haematology, Tan Tock Seng Hospital, 11 Jln Tan Tock Seng, Singapore 308433 (e-mail:

Bingwen_Eugene_Fan@ttsh.com.sg).

bophilia workup (including tests for antiphospholipid syndrome, protein $\mathrm{C}$, protein $\mathrm{S}$, antithrombin levels, and factor $V$ Leiden mutation) was negative, with von Willebrand factor (VWF) and D-dimer levels remaining persistently elevated weeks after their initial thrombotic event. Our observations suggest that the SARS-CoV-2 virus poses significant chronic immuno-thrombogenicity which may cumulate eventually in a major thrombotic event that occurs unexpectedly many weeks later in fit patients with mild or asymptomatic COVID-19 infection. Sustained prothrombotic changes in COVID-19 patients were found in a separate study, ${ }^{11}$ with increased thrombin generation, decreased plasma fibrinolysis, elevated factor VIII, VWF, and plasminogen activator inhibitor-1 (PAI-1) levels on admission and 4 months after discharge, suggestive of platelet activation and ongoing intravascular coagulation. Several other case reports highlight the phenomena of post COVID-19 arterial events as well. Notably, two case reports demonstrated the development of late, lower extremity, multilevel arterial thrombosis in a 24-year-old male and a 54-year-old male, both with nonsevere COVID-19 and without previous significant medical histories. ${ }^{6,7}$ A previously healthy 61 -year-old female with COVID-19 not requiring hospitalization presented 32 days later with fatal main pulmonary artery thrombosis discovered on autopsy. ${ }^{8}$ Although pulmonary artery thrombosis is often considered a form of venous thrombosis, as the occlusion can result from emboli arising from a deep vein thrombosis, we make a point here that (1) this will still terminate in the pulmonary arteries and not pulmonary veins and (2) that in COVID-19, pulmonary thromboses are thought to originate in the pulmonary arteries/vasculature. ${ }^{12}$ Also, a 35-year-old male with a medical history of mild-intermittent asthma and obesity published online April 15, 2021
Issue Theme Maintaining Hemostasis and Preventing Thrombosis in COVID-19-Part III; Guest Editors: Emmanuel J. Favaloro, PhD, FFSc (RCPA) and Giuseppi Lippi, MD (c) 2021. Thieme. All rights reserved. Thieme Medical Publishers, Inc., 333 Seventh Avenue, 18th Floor, New York, NY 10001, USA
DOI https://doi.org/ 10.1055/s-0041-1728717. ISSN 0094-6176. 
presented 49 days after the onset of flu-like illness not requiring hospitalization (SARS-CoV-2 antibodies were positive on day 2 of admission) with extensive thrombosis including a large left ventricle thrombus, right coronary artery thrombosis, and a middle cerebral artery thrombosis. ${ }^{9}$ In summary, these post COVID-19 cases, most of who were young and with no evident cardiovascular risk factors, developed delayed, massive arterial thromboses.

Although post thrombotic events are known to occur after acute viral infections such as influenza, ${ }^{13,14}$ where up to a third of acute myocardial infarctions are preceded by an upper respiratory infection, less is known about the longterm impact on vascular health and delayed manifestation of thrombosis after recovering from COVID-19. The classic Virchow's triad for thrombosis consists of stasis, endothelial injury, and hypercoagulable state. While hypercoagulability and stasis impact primarily on acute thrombogenesis, they may play less prominent roles in delayed arterial events observed in convalescent COVID-19 patients. Vascular underpinning of post COVID-19 thrombosis has centered on endothelial dysfunction as one of the key mechanisms. This occurs likely because of prior cytotoxic effects of SARS-CoV-2 infection and overactive immune response causing prolonged vasculitis. ${ }^{15}$ This culminates in breach of endothelial barrier integrity, exposing tissue factors and procoagulant cytokines from underlying vessels, thus triggering tissue factor driven secondary hemostasis.

Evidence for endothelial dysfunction during post COVID-19 recovery has been shown in children. Multisystem inflammatory syndrome, ${ }^{16}$ comprising post-viral myocarditis and inflammatory vasculopathy, is attributed to prior trophism of coronavirus to endothelial cells. Children with COVID-19associated chilblains of extremities have also been observed to show marked endothelial inflammation, with endothelial infection by SARS-CoV-2. ${ }^{17}$ In addition, autopsy findings of COVID-19 adult patients show severe endothelial injury associated with the presence of intracellular SARS-CoV-2, cell membrane disruption, and vascular angiogenesis. ${ }^{18}$ More recently, detailed interpretation of pathological reports has explained involvement of vascular-immune crosstalk in extensive pulmonary thrombosis, resulting in systemic microembolism which may have caused multiorgan ischemia and complications. ${ }^{15}$ All these aforementioned events may contribute to possible persistence of endothelial dysfunction, as shown by increased numbers of circulating endothelial cells (biomarker of vascular injury) and more pronounced endothelial activation markers (ICAM-1, P-selectin) in recovered COVID-19 patients compared with healthy non-COVID-19 individuals. $^{19}$

The rapid rise in COVID-19 infection exacerbated by more virulent variants of SARS-CoV-2 such as B.1.1.7, B.1.351, and $\mathrm{P} 1 / 2,{ }^{20}$ and growing numbers of COVID-19 "long haulers," is expected to cause an increased global healthcare burden. The evidence of persistent endothelial dysfunction in post COVID-19 patients, including those with either no or minimal symptoms during initial infection, raises pressing concerns about the potential long-term cardiovascular effects. Besides arterial thromboembolism, COVID-19 survivors developing endothelial dysfunction may experience chronic cardiovascular complications such as heart failure, sudden cardiac death, arrhythmias (especially atrial fibrillation), accelerated arteriosclerosis, ${ }^{21}$ pulmonary hypertension, ${ }^{22}$ and arterial aneurysms. ${ }^{23}$ Moreover, the incidence of vascular events due to endothelial dysfunction from COVID-19 in survivors is likely to be exacerbated by comorbidities such as hypertension and diabetes. Immunomodulatory therapy targeting thromboinflammation in COVID-19, ${ }^{24}$ such as tocilizumab (antiinterleukin-6 receptor monoclonal antibodies) or the use of high-dose steroid therapy, may have benefits in reducing the inflammatory cascade in COVID-19 and endotheliitis in at-risk patients, and requires further evaluation. The efficacy and safety of prolonged thromboprophylaxis in post COVID-19 survivors is dependent on an increased understanding of the pathophysiology and mechanisms of such complications. Therefore, further systematic studies on COVID-19-associated thrombosis are necessary and results from ongoing clinical trials such as the ACTIV-4 trial (NCT04498273) evaluating prolonged thromboprophylaxis following discharge are eagerly awaited.

Conflict of Interest

None declared.

\section{References}

1 Di Minno A, Ambrosino P, Calcaterra I, Di Minno MND. COVID-19 and venous thromboembolism: a meta-analysis of literature studies. Semin Thromb Hemost 2020;46(07):763-771

2 Fan BE, Ng J, Chan SSW, et al. COVID-19 associated coagulopathy in critically ill patients: a hypercoagulable state demonstrated by parameters of haemostasis and clot waveform analysis. J Thromb Thrombolysis 2021;51(03):663-674

3 Late Sequelae of COVID-19. Updated November 13, 2020. Accessed March 25, 2021 at: https://www.cdc.gov/coronavirus/2019-ncov/ hcp/clinical-care/late-sequelae.html

4 COVID-19 rapid guideline: managing the long-term effects of COVID-19 NICE guideline [NG188]. Accessed December 18 2020 at: https://www.nice.org.uk/guidance/ng188

5 Fan BE, Umapathi T, Chua K, et al. Delayed catastrophic thrombotic events in young and asymptomatic post COVID-19 patients. J Thromb Thrombolysis 2021;51(04):971-977

6 Veyre F, Poulain-Veyre C, Esparcieux A, et al. Femoral arterial thrombosis in a young adult after nonsevere COVID-19. Ann Vasc Surg 2020;69:85-88

7 Schweblin C, Hachulla AL, Roffi M, Glauser F. Delayed manifestation of COVID-19 presenting as lower extremity multilevel arterial thrombosis: a case report. Eur Heart J Case Rep 2020;4 (06):1-4

8 Del Nonno F, Colombo D, Nardacci R, Falasca L. Fatal pulmonary arterial thrombosis in a COVID-19 patient, with asymptomatic history, occurred after swab negativization. Thromb J 2021;19 (01):1

9 Ranard LS, Engel DJ, Kirtane AJ, Masoumi A. Coronary and cerebral thrombosis in a young patient after mild COVID-19 illness: a case report. Eur Heart J Case Rep 2020;4(05):1-5

10 Taquet M, Geddes JR, Husain M, et al. Six-month neurological and psychiatric outcomes in 236,379 survivors of COVID-19. medRxiv2021.01.16.21249950. Doi: 10.1101/2021.01.16.21249950 
11 von Meijenfeldt FA, Havervall S, Adelmeijer J, et al. Sustained prothrombotic changes in COVID-19 patients 4 months after hospital discharge. Blood Adv 2021;5(03):756-759

12 McGonagle D, O'Donnell JS, Sharif K, Emery P, Bridgewood C. Immune mechanisms of pulmonary intravascular coagulopathy in COVID-19 pneumonia. Lancet Rheumatol 2020;2(07): e437-e445

13 Kwong JC, Schwartz KL, Campitelli MA. Acute myocardial infarction after laboratory-confirmed influenza infection. N Engl J Med 2018;378(26):2540-2541

14 Lippi G, Sanchis-Gomar F. Cardiac troponin elevation in patients with influenza virus infections. Biomed J 2020:S2319-4170(20) 30088-3

15 McGonagle D, Bridgewood C, Ramanan AV, Meaney JFM, Watad A. COVID-19 vasculitis and novel vasculitis mimics. Lancet Rheumatol 2021;3(03):e224-e233

16 Jiang L, Tang K, Levin M, et al. COVID-19 and multisystem inflammatory syndrome in children and adolescents. Lancet Infect Dis 2020;20(11):e276-e288

17 Colmenero I, Santonja C, Alonso-Riaño M, et al. SARS-CoV-2 endothelial infection causes COVID-19 chilblains: histopathological, immunohistochemical and ultrastructural study of seven paediatric cases. Br J Dermatol 2020;183(04): 729-737
18 Ackermann M, Verleden SE, Kuehnel M, et al. Pulmonary vascular endothelialitis, thrombosis, and angiogenesis in COVID-19. N Engl J Med 2020;383(02):120-128

19 Chioh FW, Fong SW, Young BE, et al. Convalescent COVID-19 patients are susceptible to endothelial dysfunction due to persistent immune activation. eLife 2021;10:e64909

20 Lippi G, Henry B. How will emerging SARS-CoV-2 variants impact herd immunity? SSRN 2021https://ssrn.com/abstract=3791910. Doi: $10.2139 /$ ssrn.3791910

21 Becker RC. Anticipating the long-term cardiovascular effects of COVID-19. J Thromb Thrombolysis 2020;50(03):512-524

22 Dhawan RT, Gopalan D, Howard L, et al. Beyond the clot: perfusion imaging of the pulmonary vasculature after COVID-19. Lancet Respir Med 2021;9(01):107-116

23 Pick JM, Wang S, Wagner-Lees S, et al. Coronary artery aneurysms are more common in post COVID-19 multisystem inflammatory syndrome in children (MIS-C) than pre-pandemic Kawasaki disease. November 17, 2020;142(Suppl 3): Abstracts From the American Heart Association's 2020 Scientific Sessions

24 Bikdeli B, Madhavan MV, Gupta A, et al; Global COVID-19 Thrombosis Collaborative Group. Pharmacological agents targeting thromboinflammation in COVID-19: review and implications for future research. Thromb Haemost 2020;120(07): 1004-1024 